in the contralateral same gyrus.

2) $0.5 \mathrm{~mol}$ INAH evoked, spike \& dome waves with a latent period of 30 minutes persisting for 90 minutes. But $1 \mathrm{~mol}$ glucose, and 0.01-1 mol metrasol solution produced no such changes.

3) Either GABA or INAH within dorsal medial nuclei often showed long lasting spindle burst type recruiting waves.

It is suggested from the above observations that certain chemical substances produce spike $\&$ dome wave from intralaminar nuclei of cats.

\title{
100. Effect of EEG Arousal upon Photic Driving
}

\section{Ziro Kaneko, Yasuhiko Hidaka, Yasuo Hishikawa, Akira Shimizu, Hideaki Kameda AND Kiyoshi MiYAZAKI \\ Department of Neuropsychiatry, Osaka University Medical School}

Acute cat-preparations, immobilized with succynylcholine and maintained on artificial respiration, were employed. Eyelids were kept widely open and $1 \%$ solution of atropine was dropped in the eyes in order to abolish pupillary reflex.

Cortical photic responses driven by repeated rhythmic flicker stimulation (Buffington photic stimulator, $1-30 \mathrm{c} / \mathrm{s}$ ) were recorded. An ink-writing recorder and an automatic frequency analyser was used in this study.

The photic driving responses (fundamentals, harmonics and subharmonics) were modified by arousal stimulation; sensory stimulation (tactile and auditory), sciatic nerve stimulation or electrical stimulation of the midbrain reticular formation $(1 \mathrm{msec}, 100 \mathrm{c} / \mathrm{s}$, square wave).

From these results, the authors postulated that photic driving was affected by arousal function, and variable types of photic driving might be classified from the viewpoint of arousal level. In clinical cases showing asymmetric photic driving, this postulation might be helpful in diagnosing which side of arousal function is lower.

\section{Analysis of Amino Acids in Brain with lon Exchange Resin}

Dennosuke Jinnai, Yasuhisa Yamamoto, Sakae Matano, Junji Kasahara AND Akitane MoRI

Department of Surgery and Neurological Surgery, Okayama

University School of Medicine

Analysis of free amino acids in the brain was made by Moore's improved method, using ion exchange resin "Amberlite IR-120" with columns of $150 \mathrm{~cm}$. and $50 \mathrm{~cm}$. in length. 